Check for updates

Cite this: RSC Adv., 2018, 8, 8240

\title{
p-Hexafluoroisopropanol phenyl functionalized graphene for QCM based detection of dimethyl methylphosphonate, a simulant of the nerve agent sarin
}

\author{
Mahabul Shaik, ${ }^{a}$ V. Kameswara Rao, (D) ${ }^{* a}$ G. V. Ramana, ${ }^{\text {b M. Halder, }}{ }^{\text {a P. K. Gutch, }}{ }^{a}$ \\ P. Pandey and R. Jain ${ }^{\mathrm{c}}$
}

The detection of DMMP (dimethyl methylphosphonate, a simulant of nerve agent sarin) was performed by using $p$-hexafluoroisopropanol phenyl (HFIPP) functionalized graphene (GR) via hydrogen bond interactions. For this, the HFIPP moiety was covalently functionalized on the surface of GR by a diazo reaction. The HFIPP-GR film-modified QCM electrodes were fabricated and their sensing characteristics towards DMMP were investigated. The proposed sensor showed good response towards sensing DMMP vapor at room temperature. In order to see the effect of HFIPP derivatives on DMMP vapor sensing, a comparative study was also conducted with unfunctionalized graphene. The sensitivity and detection limit of the HFIPP-GR sensor against DMMP vapors were $12.24 \mathrm{~Hz} \mathrm{ppm}^{-1}$ and $150 \mathrm{ppb}$ respectively. The HFIPP-GR coated sensors showed good selectivity towards sensing DMMP vapors when compared with common organic vapors.

Received 9th November 2017 Accepted 19th January 2018

DOI: $10.1039 / c 7 r a 12272 a$

rsc.li/rsc-advances and capillary electrophoresis coupled with flame photometric and conductivity detection, ${ }^{9-11}$ were routinely employed to analyze the nerve agents in environmental and biological samples. Though these methods have very high sensitivity and reliability, these techniques are time consuming, require expensive instrumentation and also highly trained personnel, which make these techniques unsuitable for on-site analysis. AChE inhibition biosensors ${ }^{\mathbf{1 2 - 1 4}}$ and optical methods such as fluorogenic and chromogenic sensors ${ }^{15-19}$ were widely explored in recent times. Though inhibition biosensors are highly sensitive, the stability of enzymes and interference with other OPCs and metal ions hinder their applications in selective sensors for nerve agents. Most of the optical sensors reported were successful in detecting the nerve agents present in the liquid samples at lower concentrations. But, the detection of the nerve agents in vapor and aerosol forms is crucial for practical sensors. Surface acoustic wave (SAW) or quartz crystal microbalance (QCM) sensors and chemiresistive gas sensors could be a reliable and promising alternative to routine methods because of their simplicity, low power requirement, ease of use and field deployable with high sensitivity and selectivity. ${ }^{\mathbf{2 0 - 2 5}}$

Chemical warfare agents, especially nerve agents, are strong hydrogen-bond basic compounds. Hence, strongly hydrogenbond acidic polymers for sensing vapors of strong hydrogenbond basic nerve agents and explosives were introduced few decades back. Initially, these polymers were of interest for gaining high sensitivity to nerve agents using acoustic wave sensors. In such sensors, the hydrogen-bond acidic polymer 
acted as the sorbent layer on the crystal surface and interacted with the vapors of strongly hydrogen-bond basic organophosphorus compounds via hydrogen bonding. ${ }^{26}$ These interactions promoted sorption of these vapors into the polymer film coated on the crystal surface, which increased the sensor response. The property of hydrogen-bond acidity was incorporated by fluorinated alcohol or fluorinated phenol functional groups into the polymer structure. Though there was an extensive study on sensing vapors of CWAs using hydrogen-bond acidic polymers, the stability of the polymers and swelling in the presence of humidity limits their applications in the realization of portable sensors for CWAs.

Recent development of nanotechnology has created huge potential to build highly sensitive, low cost, portable sensors with low power consumption. The extremely high surface-tovolume ratio is ideal for gas molecules adsorption. Therefore, gas sensors based on nanomaterials, such as carbon nanotubes (CNTs) and graphene have been investigated widely for the detection of CWAs. ${ }^{27-30}$ More recently, investigations on sensors based on hydrogen-bond acidic moieties such as hexafluoroisopropanol (HFIP) functionalized nanomaterials have been explored for detecting dimethyl methylphosphonate (DMMP). In contrast with bare nanomaterials, decoration or functionalization of nanomaterials with HFIP derivatives can greatly improve sensitivity and selectivity of the sensors. ${ }^{31-34} \mathrm{~F}$. Wang et al. reported sub-ppm chemiresistive detection of DMMP using HFIP-functionalized polythiophene. ${ }^{32}$ QCM based sensor were fabricated for detecting DMMP by functionalizing the mesoporous inorganic host (SBA-15) with HFIP functional moieties. ${ }^{35,36}$ Hence, it can be predicted that HFIP derivative can be widely applied as powerful intermediate sensing material having excellent sensitivity and selectivity for detecting nerve agents or its simulant, dimethyl methylphosphonate (DMMP), due to selective accumulation of analyte molecules on HFIP substituents via hydrogen bonding. The application of nanomaterials as sorbent layer also circumvents the problems associated with using polymers such as swelling and stability of the polymers during their operation.

Here, we prepared graphene- $p$-hexafluoroisopropanol phenyl (HFIPP-GR) hybrids via in situ diazonium reaction of $p$ hexafluoroisopropanol aniline (HFIPA) with graphene (GR) and utilized this material for sensing DMMP by using QCM based transducers.

\section{Experimental section}

\subsection{Materials and reagents}

Few layered argon plasma treated graphene (GR) used in this study was received from $\mathrm{M} / \mathrm{s}$ Nanospan India Pvt Ltd, India. $p$ Hexafluoroisopropanol aniline (HFIPA) was purchased from M/ s ClearSynth India Pvt Ltd. Isoamylnitrite, 1,2 dichlorobenzene, acetonitrile were purchased from Aldrich and used as received.

\subsection{Preparation of sensing materials}

$p$-Hexafluoroisopropanol phenyl (HFIPP) moiety was grafted on graphene surface via diazo chemistry. ${ }^{37,38}$ In a typical synthesis,
$20 \mathrm{mg}$ of GR was dispersed in 1,2-dichlorobenzene using ultrasonication for $1 \mathrm{~h}$. To this $10 \mathrm{~mL}$ of acetonitrile containing $200 \mathrm{mg}$ of HFIPA was added drop wise and stirred for 10 minutes in nitrogen atmosphere. To this mixture, $0.2 \mathrm{~mL}$ of isoamylnitrite was added quickly and stirred for $24 \mathrm{~h}$ at $70{ }^{\circ} \mathrm{C}$ in dark. Then the reaction mixture was allowed to cool to room temperature naturally followed by vacuum filtration using nylon-6 (pore size of $0.2 \mu \mathrm{m}$ ) membrane. The residue was washed with DMF until the color of the filtrate becomes colorless; finally it was washed with ethanol and dried at $70^{\circ} \mathrm{C}$ for $4 \mathrm{~h}$ under vacuum. The reaction scheme was depicted in Fig. 1. The obtained material was labeled as HFIPP-GR.

\subsection{Characterization of sensing materials}

The morphology of as received graphene was studied by high resolution transmission electron microscopy (HRTEM) (FEI, Technai $\mathrm{G}^{2} \mathrm{~S}$-Twin) at an operating voltage of $200 \mathrm{kV}$. Energydispersive X-ray spectroscopy was recorded with EDAX connected to SEM. Thermal gravimetric analysis was conducted using Perkin-Elmer Instruments.

\subsection{Gas sensing measurements}

The gas sensing characteristics of the prepared materials were studied using QCM based transducers. $8 \mathrm{MHz}$ AT-cut gold coated QCM crystals were purchased from $\mathrm{CH}$ instruments, USA. Drop-drying method was used for depositing the sensing materials (GR and HFIPP-GR) onto the surface of the gold coated QCM crystal. A small known amount of GR or HFIPP-GR was dispersed in $N, N^{\prime}$-dimethylformamide $\left(0.5 \mathrm{mg} \mathrm{mL}^{-1}\right)$ by ultrasonication for about 30 minutes, and subsequently a small volume of the obtained dispersion was placed carefully on the centre of the QCM electrodes using a micro-syringe. Then, the coated electrodes were dried at $80^{\circ} \mathrm{C}$ for $2 \mathrm{~h}$ under vacuum to obtain thin film of sensing materials on electrode surface. The same procedure was followed to form thin films of sensing materials on electrode surface, unless otherwise stated.

In this study, DMMP was used as a nerve agent simulant for G-type nerve agents because of the common occurrence of the $\mathrm{P}=\mathrm{O}, \mathrm{P}-\mathrm{CH}_{3}$ and $\mathrm{P}-\mathrm{OCH}_{3}$ functional groups, which also exist in the nerve agents and less toxicity of DMMP.

Fig. 2 illustrates the schematic diagram of QCM based sensing assembly. For gas sensing measurements, dry nitrogen gas was bubbled through DMMP reservoir, and the obtained DMMP vapors (at saturated vapor pressure) were

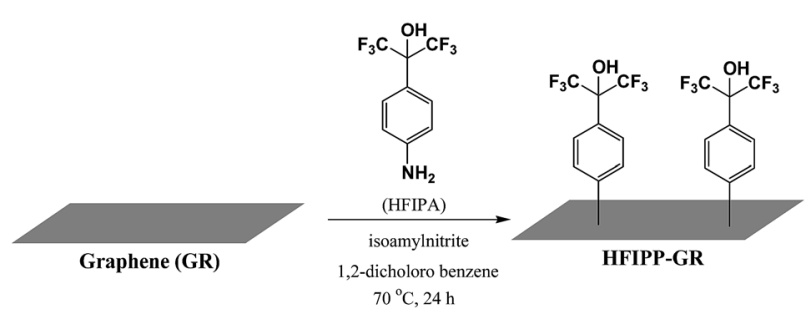

Fig. 1 Preparation of $p$-hexafluoroisopropanol phenyl functionalized graphene. 


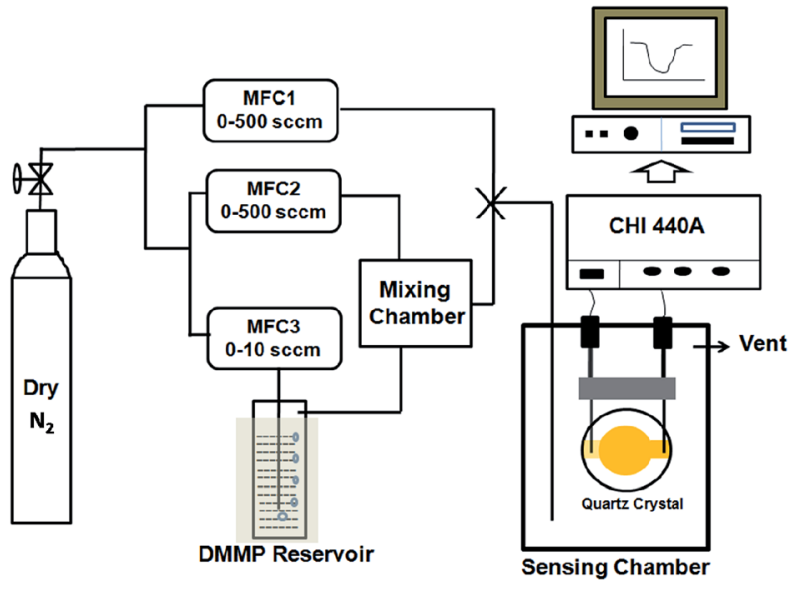

Fig. 2 Schematic gas sensing assemblies of QCM based sensors for the detection of DMMP vapors.

diluted with nitrogen gas to get desired concentrations of DMMP. The total flow rate of the test gas and the diluent gas

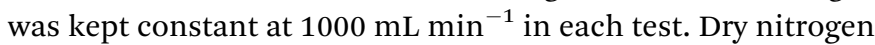
was used as diluent gas and all sensing experiments were performed at room temperature. The saturated vapour pressure of DMMP was calculated using Antoine Equation. ${ }^{39}$ The desired concentration of the DMMP was subjected into the chamber, where the coated QCM crystal was present, and the change in frequency of the crystal due to exposure of DMMP was measured as $\Delta F(\mathrm{~Hz})$.

\section{Result and discussion}

\subsection{Preparation and characterization of sensing materials}

The HR-TEM images of as received argon plasma treated graphene was shown in Fig. 3. The received graphene sample contains few layers (up to 4 layers) of graphene, which is ideal for sensitive detection of gaseous analytes.

The quantitative estimation for the functionalization degree of graphene can be performed through TGA by measuring the mass loss accompanied with functional moieties removed from the graphene in an inert atmosphere by heating. The TGA analysis was performed by heating the samples, HFIPP-GR and $\mathrm{GR}$, from room temperature to $800^{\circ} \mathrm{C}$ in a nitrogen atmosphere with a ramp of $20^{\circ} \mathrm{C} \mathrm{min}^{-1}$. As shown in Fig. 4, the mass loss of $14 \%$ and $32 \%$ was observed in the TGA experiment for GR and

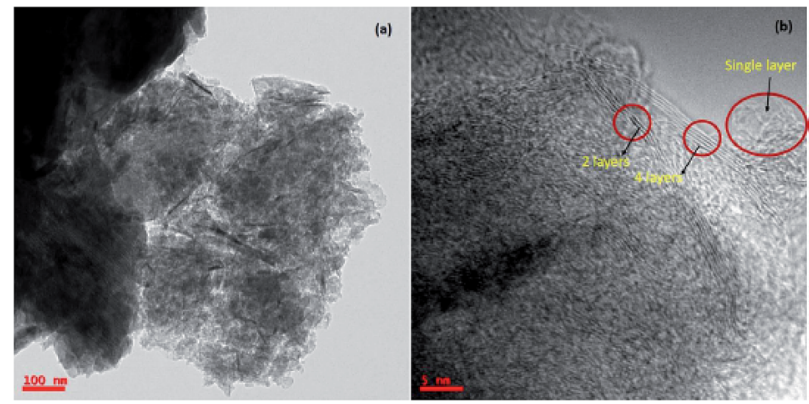

Fig. 3 HR-TEM images of argon plasma treated graphene.

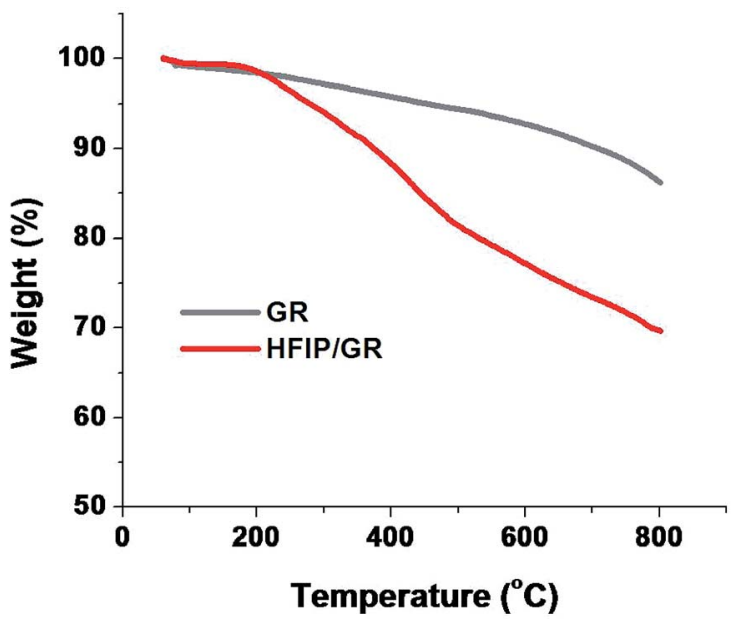

Fig. 4 TGA of GR and HFIPP-GR.

HFIPP-GR respectively. For HFIP-GR, the sharp mass loss was observed at $200-450{ }^{\circ} \mathrm{C}$. The mass loss of GR may due to the adsorbed moisture as well as the defects in the graphene. Where as, in the case of HFIP-GR, the excess $18 \%$ mass loss can be attributed to the functionalization of GR, which suggests that one $p$-hexafluoroisopropanol moiety was functionalized per 76 carbon atoms on the basis of all the weight loss only depended on the functional components. From this the amount of fluorine present in the HFIP-GR can be estimated to $8.4 \mathrm{wt} \%$.

The EDS analysis was performed to ensure the functionalization on graphene and also to know the composition of elements (carbon, oxygen and fluorine) present in the sensing materials. The EDS spectrum of GR and HFIPP-GR was presented in Fig. 5(a) and (b) respectively, and the corresponding elemental composition of the material was shown in inlet tables of Fig. 5. The presence of fluorine in HFIPP-GR was indicated the functionalization of graphene with fluorine containing functional moiety. From EDS, it was also clear that the fluorine concentration in HFIPP-GR was around $8.18 \mathrm{wt} \%$, which is in consistent with the estimation of fluorine by TGA (i.e., $8.4 \mathrm{wt} \%$ ). The fluorine content in the functionalized material was also in consistent with the earlier reported methods. ${ }^{37}$

\subsection{DMMP sensing characteristics of HFIPP-GR}

3.2.1 Effect of film thickness. The HR-TEM images and nitrogen adsorption/desorption analysis indicates the porous nature of the sensing material. The sensor characteristics can be improved by optimizing the thickness of the sensing material film on the crystal surface. For these different volumes $(0,2$, $3.5,5,7,10$ and $15 \mu \mathrm{L})$ of dispersion of sensing material $(0.5 \mathrm{mg}$ of HFIPP-GR in $1 \mathrm{~mL}$ of DMF) was placed on QCM crystal and drop-dried. As the volume of the dispersion increases, the thickness of the film increases and there by the oscillating frequency of the crystal decreases. The change in oscillating frequency of the crystal as a function of volume of the dispersion was plotted in Fig. 6(a).

The oscillating frequencies of the crystals coated with different amounts of HFIPP-GR were measured by placing 
a)

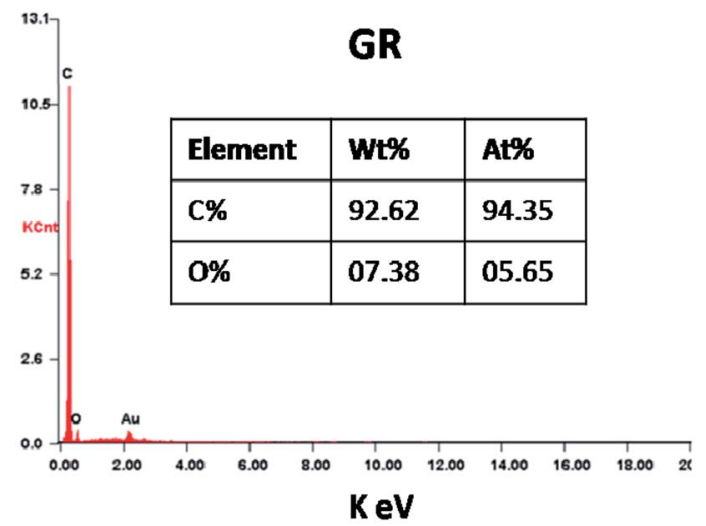

b)

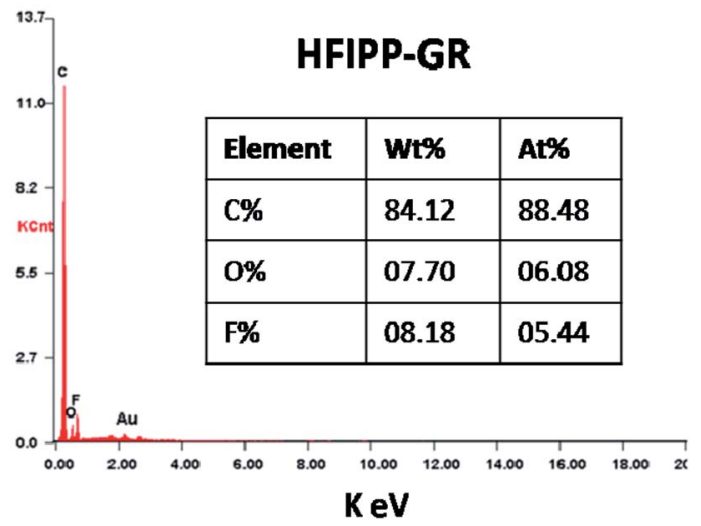

Fig. 5 Energy dispersive X-ray spectrum of (a) GR and, (b) HFIPP-GR.

in sensing chamber and purging dry nitrogen gas. Then, the coated crystals were exposed to $5 \mathrm{ppm}$ of DMMP for 5 minutes and the corresponding changes in frequencies were measured. The change in frequencies due to the exposure of $5 \mathrm{ppm}$ of DMMP were plotted against volume of HFIPP-GR and shown in Fig. 6(b). From Fig. 6(b), it was clear that the sensor response increases as the thickness (volume of coating) of the HFIPP-GR film increased from 0 to $10 \mu \mathrm{L}$, and then became saturated at a thickness greater than $10 \mu \mathrm{L}$. Therefore, for the sensitive detection of DMMP vapors, we chose HFIPP-GR film obtained by drop-drying $10 \mu \mathrm{L}$ of the dispersion.

3.2.2 Response-recovery characteristics. The typical response-recovery curve of HFIPP-GR coated crystal was shown in Fig. 7, after attaining a stable baseline frequency, $5 \mathrm{ppm}$ of DMMP vapors were introduced into the chamber, where the coated crystal was present. Due to the adsorption of the DMMP vapors on sensor surface, the oscillating frequency of the sensor decrease. After attaining a saturated frequency value, the chamber was purged with pure $\mathrm{N}_{2}$ to allow the sensor to return to its initial condition. The responses were recorded until the signal variations were less than $1 \mathrm{~Hz} \mathrm{~min}^{-1}$ for at least three successive minutes. The change in frequency of HFIPP-GR coated crystal due to the exposure of $5 \mathrm{ppm}$ DMMP vapor is $71 \pm 4 \mathrm{~Hz}$.

Furthermore, the HFIPP-GR coated sensor was exposed to various concentrations of DMMP vapors. It is obvious that as the concentration of the DMMP increased, the frequency shift was also increased. It exhibits good and fast response, and negligible baseline drift. The response of HFIPP-GR coated sensor is shown in Fig. 8(a) by plotting the frequency shifts $(\mathrm{Hz})$ against the concentrations of DMMP vapor (in ppm). Fig. 8(a) shows that the frequencies of the HFIPP-GR coated QCM sensor can return to about $80 \%$ of original values after desorption processes (purging nitrogen for 10 minutes). The reason for long recovery times was ascribed to the strong interaction between DMMP molecules and HFIPP-GR films. The sensitivity of the sensor is as high as $12.24 \mathrm{~Hz} \mathrm{ppm}^{-1}$. The limit of detection of HFIPP-GR coated QCM sensor at signal to noise ratio of 3 is around $150 \mathrm{ppb}$. For comparison GR coated QCM crystal was also exposed to the various concentrations of the DMMP and the obtained response was also shown in Fig. 8, the sensitivity of this sensor was $3.85 \mathrm{~Hz}$ $\mathrm{ppm}^{-1}$ of DMMP. The response of the sensor coated with GR was much lower than that of HFIPP-GR coated sensor, indicating the decoration of graphene with HFIP derivatives enhanced many folds increase in the response towards sensing DMMP vapors. The higher response of HFIPP-GR, on comparison with GR was attributed to the strong hydrogen bonding between HFIP moiety and DMMP vapor.
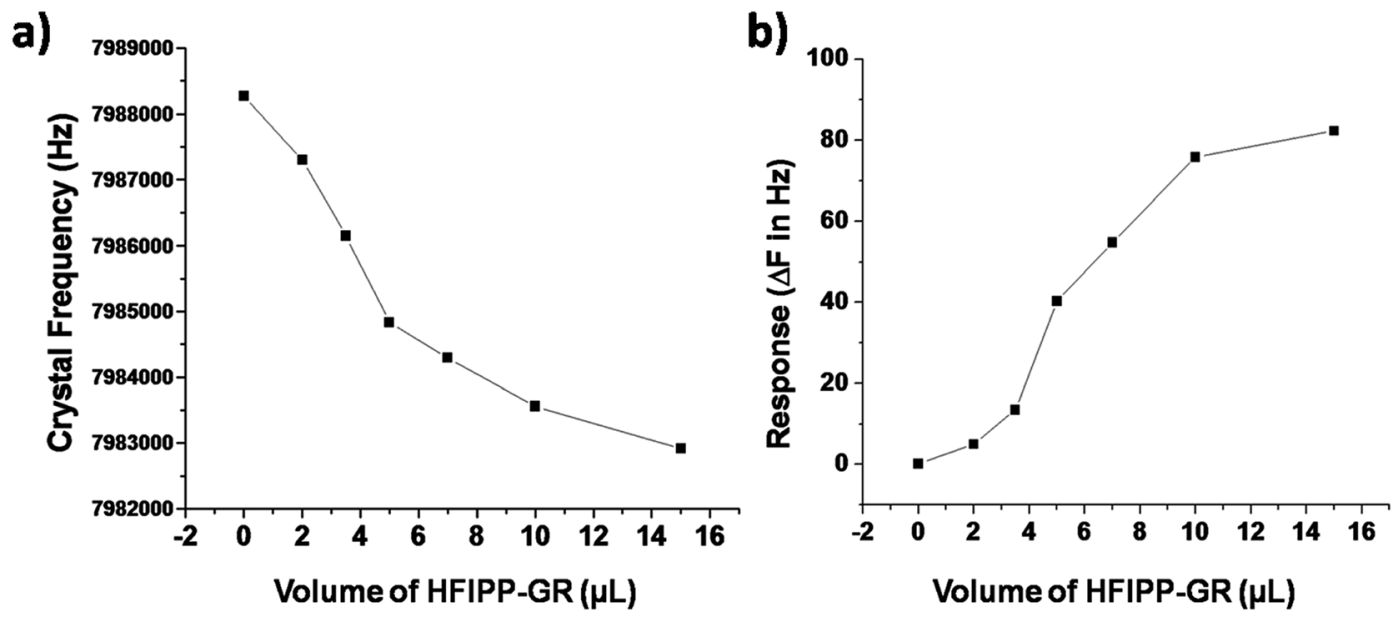

Fig. 6 Effect of thickness of the HFIPP-GR film on (a) crystal frequency and (b) response of the sensor for 5 ppm of DMMP. 


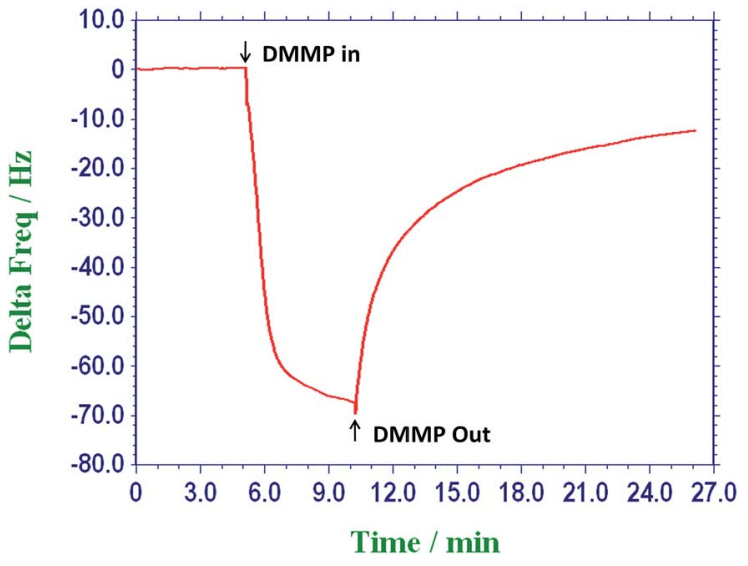

Fig. 7 Response-recovery curves of HFIPP-GR coated QCM sensor when exposed to 5 ppm DMMP vapors.

3.2.3 Reproducibility studies. To investigate the reproducibility of the sensor, the HFIPP-GR coated QCM sensor was repeatedly exposed to DMMP (5 ppm) for seven times and the time dependent frequency shifts were recorded (Fig. 9). After each exposure, $\mathrm{N}_{2}$ was purged through the chamber for 10

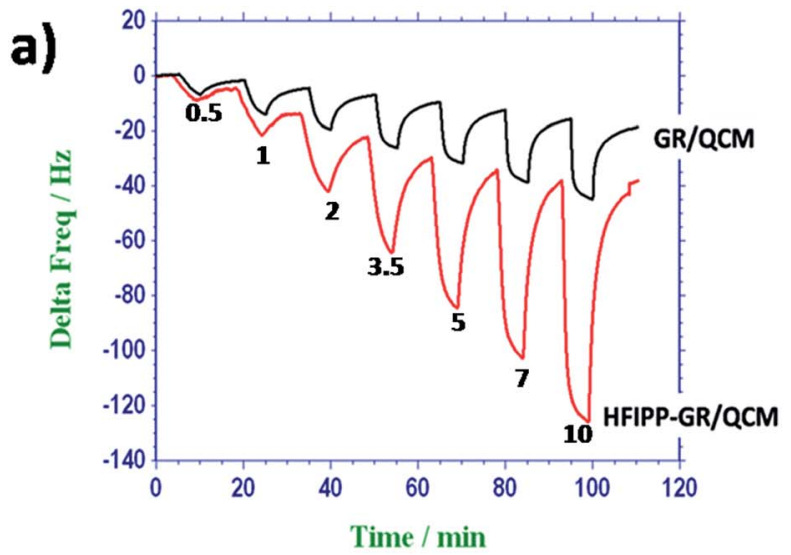

b)

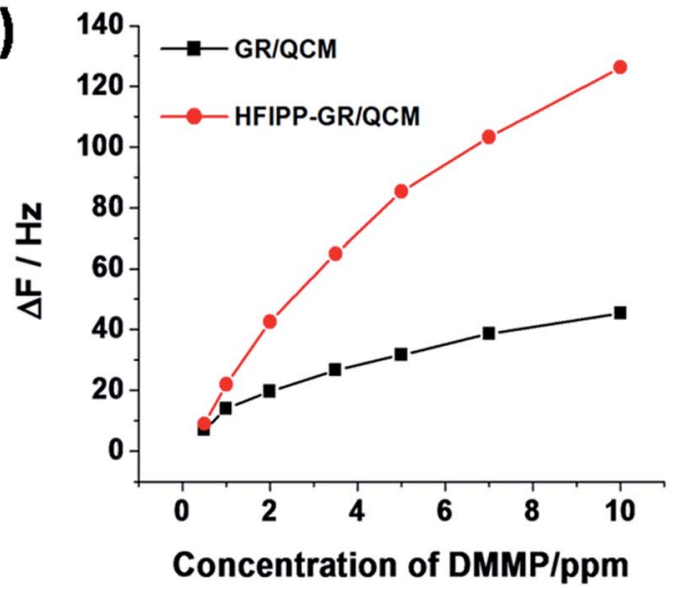

Fig. 8 (a) DMMP sensing characteristics of HFIPP-GR/IDE sensor when exposed to various concentrations (the concentrations of DMMP in ppm were shown on the peaks); (b) response of sensor as a function of concentration of DMMP.

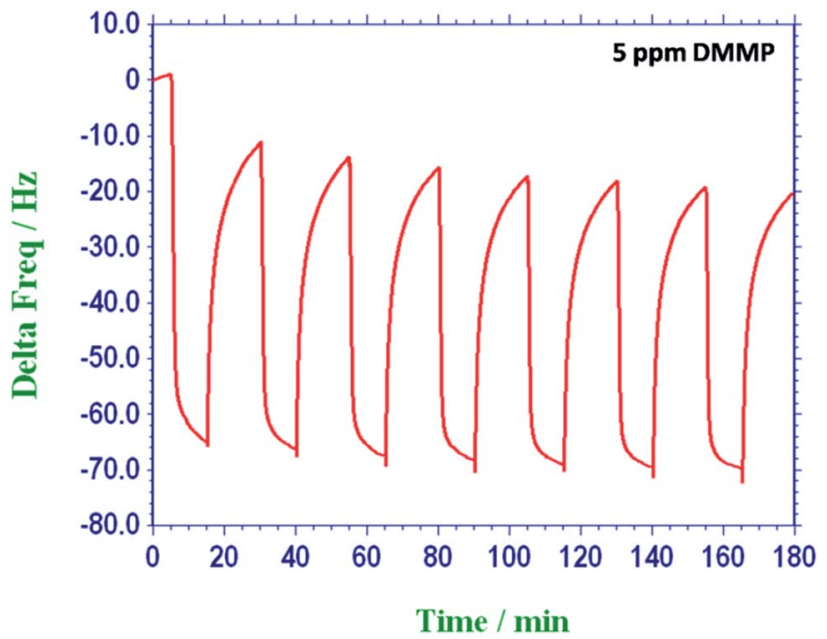

Fig. 9 Reproducibility studies of HFIPP-GR coated QCM sensor exposed to $5 \mathrm{ppm}$ of DMMP.

minutes. It was found that the HFIPP-GR modified sensor showed similar results on repetition, whereas there was high response during first cycle of exposure of DMMP. This may be due to availability all active sites for the adsorption of DMMP vapors. After first cycle, the response of the sensor was almost constant for DMMP up to studied number of cycles.

3.2.4 Sensitivity to interferants. The HFIPP-GR coated QCM sensor was also tested against several conventional volatile organic vapors, which may act as potential interferences including ethanol, benzene, acetone, toluene, dichloromethane (DCM) and dimethylsulfoxide (DMSO). Each analyte at a concentration of $5 \%$ saturated vapor concentration was generated by conventional bubble method, where a carrier gas $\left(\mathrm{N}_{2}\right)$ was passed through analyte liquids at a flow rate of 25 $\mathrm{mL} \min ^{-1}$ and the obtained vapors were diluted with $\mathrm{N}_{2}$ to get

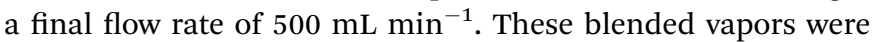
transported into the test chamber. The response of the DMMP was normalized to 1 and the responses of the remaining

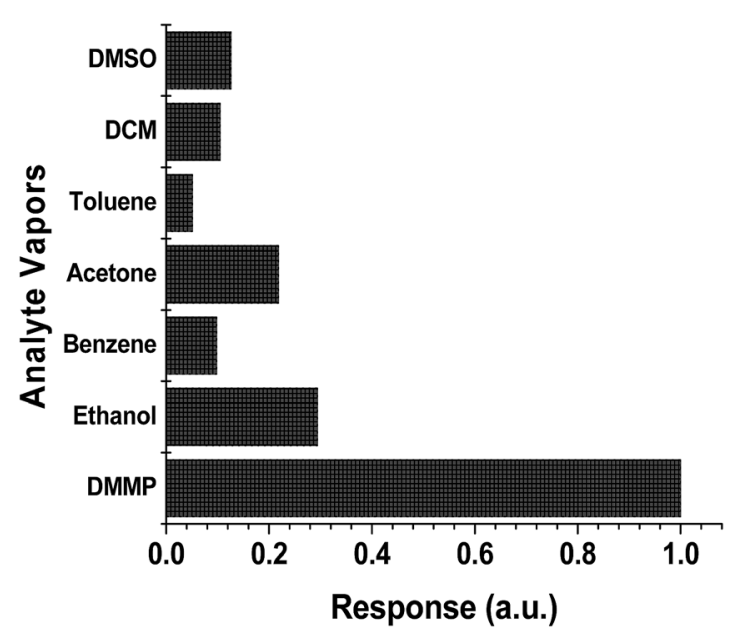

Fig. 10 Response of HFIPP-GR coated QCM sensor to various analyte vapors each at a concentration of $5 \%$ of saturated vapour concentration at $25^{\circ} \mathrm{C}$. 
analytes were calculated with respect to DMMP response. Test results are presented in Fig. 10. It shows that the sensor is more sensitive to DMMP vapor than to the interferences at 5\% of saturated vapor concentrations. Good selectivity of the HFIPPGR coated QCM sensor was achieved. The reason for the good selectivity was the strong hydrogen bond acidic and basic interactions between DMMP and the HFIP derivative, leading to the highest response for HFIPP-GR coated QCM sensor.

\section{Conclusions}

In summary, the HFIPP-GR film-modified gold coated QCM electrodes were fabricated and their sensing characteristics towards DMMP, a simulant of chemical warfare agent sarin, were investigated. The proposed QCM based sensors showed good response towards sensing DMMP vapors at room temperature. In order to see the effect of HFIP derivatives on sensing DMMP vapors, a comparative study was also conducted with as-received graphene. The graphene functionalized with HFIP derivatives showed many fold increase in response towards sensing DMMP vapors. The excellent characteristics of the sensors were due to the presence of HFIP derivative decorated on material having surface to volume i.e. graphene. The sensitivities of HFIPP-GR sensor is $12.24 \mathrm{~Hz} \mathrm{ppm}^{-1}$ against DMMP vapors. The HFIPP-GR coated sensors showed good selectivity towards sensing DMMP vapors when compared with common organic vapors. As the HFIPP-GR is stable material, by using this sensitive, selective and miniaturized devices can be fabricated to detect chemical warfare agents at room temperatures.

\section{Conflicts of interest}

There are no conflicts to declare.

\section{Acknowledgements}

The authors are thankful to the Director, DRDE for giving permission to publish. The authors are also thankful to Dr G.K. Prasad and Mrs Tara Yadav for their help in the characterization of the sensing materials.

\section{Notes and references}

1 G. Liu and Y. Lin, Electrochem. commun., 2005, 7, 339-343.

2 F. Worek, M. Koller, H. Thiermann and L. Szinicz, Toxicology, 2005, 214, 182-189.

3 D. H. Moore, J. Physiol., 1998, 92, 325-328.

4 L. Szinicz, Toxicology, 2005, 214, 167-181.

5 C. E. Kientz, J. Chromatogr. A, 1998, 814, 1-23.

6 C. E. A. M. Degenhardt-Langelaan and C. E. Kientz, J. Chromatogr. A, 1996, 723, 210-214.

7 B. A. Eckenrode, J. Am. Soc. Mass Spectrom., 2001, 12, 683-689. 8 W. R. Creasy, A. A. Rodríguez, J. R. Stuff and R. W. Warren, J. Chromatogr. A, 1995, 709, 333-344.

9 S. A. Oehrle and P. C. Bossle, J. Chromatogr. A, 1995, 692, 247-252.

10 E. W. J. Hooijschuur, C. E. Kientz and U. A. T. Brinkman, J. Chromatogr. A, 2001, 928, 187-199.
11 T. E. Rosso and P. C. Bossle, J. Chromatogr. A, 1998, 824, 125-134.

$12 \mathrm{~J}$. Anzai, Biosensors for the detection of op nerve agents, Handbook of toxicology of chemical warfare agents, 2009, pp. 837-846.

13 C. S. Pundir and N. Chauhan, Anal. Biochem., 2012, 429, 19-23. 14 E. A. Songa and J. O. Okonkwo, Talanta, 2016, 155, 289-304. 15 G. Yue, S. Su, N. Li, M. Shuai, X. Lai, D. Astruc and P. Zhao, Coord. Chem. Rev., 2016, 311, 75-84.

16 M. Gupta and H. Lee, Sens. Actuators, B, 2017, 242, 977-982.

17 D. R. Goud, D. Pardasani, V. Tak and D. K. Dubey, RSC Adv., 2014, 4, 24645-24648.

18 E. Climent, M. Biyikal, K. Gawlitza, T. Dropa, M. Urban, A. M. Costero, R. Martínez-Máñez and K. Rurack, Sens. Actuators, B, 2017, 246, 1056-1065.

19 H.-S. So, S. Angupillai and Y.-A. Son, Sens. Actuators, B, 2016, 235, 447-456.

20 B.-S. Joo, J.-S. Huh and D.-D. Lee, Sens. Actuators, B, 2007, 121, 47-53.

21 D. Matatagui, J. Martí, M. J. Fernández, J. L. Fontecha, J. Gutiérrez, I. Gràcia, C. Cané and M. C. Horrillo, Sens. Actuators, B, 2011, 154, 199-205.

22 B. Minot, C. Frenois, S. Besnard, J. Bordet, N. Martins and F. Periera, Procedia Eng., 2016, 168, 423-427.

23 X. Ji, W. Yao, J. Peng, N. Ren, J. Zhou and Y. Huang, Sens. Actuators, B, 2012, 166-167, 50-55.

24 Q. Zheng, Y.-C. Fu and J.-Q. Xu, Procedia Eng., 2010, 7, 179-184.

25 R. K. Saini, L. P. Bagri and A. K. Bajpai, New Pesticides and Soil Sensors, 2017, pp. 519-559.

26 J. W. Grate, Chem. Rev., 2008, 108, 726-745.

27 K. Kim, O. G. Tsay, D. A. Atwood and D. G. Churchill, Chem. Rev., 2011, 111, 5345-5403.

28 S. M. Kanan, O. M. El-Kadri, I. A. Abu-Yousef and M. C. Kanan, Sensors, 2009, 9, 8158-8196.

29 T. Zhang, S. Mubeen, N. V. Myung and N. A. Deshusses, Nanotechnology, 2008, 19, 332001-332014.

30 L. S. Fifield and J. W. Grate, Carbon, 2010, 48, 2085-2088.

31 A. B. Kanu, P. E. Haigh and H. H. Hill, Anal. Chim. Acta, 2005, 553, 148-159.

32 F. Wang, H. Gu and T. M. Swager, J. Am. Chem. Soc., 2008, 130, 5392-5393.

33 L. Kong, J. Wang, T. Luo, F. Meng, X. Chen, M. Lia and J. Liu, Analyst, 2010, 135, 368-374.

34 Y. Wang, Z. Wang, N. Hu, L. Wei, D. Xu, H. Wei, E. S.-W. Kong and Y. Zhang, J. Nanosci. Nanotechnol., 2011, 11, 4874-4881.

35 Q. Zheng, Y. Zhu, J. Xu, Z. Cheng, H. Li and X. Li, J. Mater. Chem., 2012, 22, 2263-2270.

36 H. Li, Q. Zheng, J. Luo, Z. Cheng and J. Xu, Sens. Actuators, B, 2013, 187, 604-610.

37 L. Kong, J. Wang, X. Fu, Y. Zhong, F. Meng, T. Luo and J. Liu, Carbon, 2010, 48, 1262-1270.

38 L. Yang, Q. Han, Y. Pan, S. Cao and M. Ding, Funct. Mater. Lett., 2014, 7, 1450043.

39 R. H. Perry, D. W. Green and J. O. Maloney, Perry's Chemical Engineers Handbook, McGraw-Hill, New York, 6th edn, 1984. 